\title{
reciam muc
}

Revista cientifica de investigación actualización del mundo de las ciencias

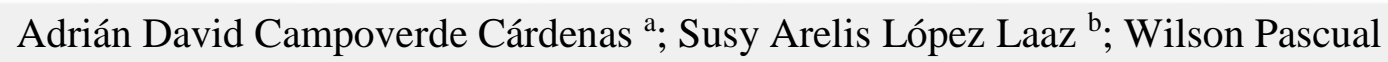

Correa Quinto ${ }^{\mathrm{c}}$; Jorge Daniel Cárdenas Rodríguez ${ }^{\mathrm{d}}$

Complicaciones de la cirugía laparoscópica

Complications of laparoscopic surgery

Revista Científica de Investigación actualización del mundo de las Ciencias. Vol. 3 núm., 2, abril, ISSN: 2588-0748, 2018, pp. 597-613

DOI: $10.26820 /$ reciamuc/3.(2).abril.2019.597-613

URL: $\underline{\text { http://reciamuc.com/index.php/RECIAMUC/article/view/355 }}$

Código UNESCO: 3205 Medicina Interna

Tipo de Investigación: Artículo de Revisión

(C) RECIAMUC; Editorial Saberes del Conocimiento, 2019

Recibido: $15 / 01 / 2019$

Aceptado: 07/02/2019

Publicado: 01/04/2019

Correspondencia: adccardenas@icloud.com

a. Médico; Investigador Independiente; Guayaquil, Ecuador; adccardenas@icloud.com

b. Médico; Investigador Independiente; Guayaquil, Ecuador; sllmed 992@ hotmail.com

c. Médico; Investigador Independiente; Guayaquil, Ecuador; willy_2293@ hotmail.com

d. Médico; Investigador Independiente; Guayaquil, Ecuador; docardenas3@gmail.com 


\section{Complicaciones de la cirugía laparoscópica}

Vol. 3, núm. 2., (2019)

Adrián David Campoverde Cárdenas; Susy Arelis López Laaz; Wilson Pascual Correa Quinto; Jorge Daniel Cárdenas Rodríguez

\section{RESUMEN}

La cirugía laparoscópica constituye hoy en día uno de los procedimientos más usados en todo el mundo por sus múltiples beneficios, no obstante, este procedimiento no se encuentra exento de presentar complicaciones. El objetivo fundamental de este estudio es plasmar las complicaciones más importantes de la cirugía laparoscópica. El diseño de investigación que se llevó a cabo es de tipo documental o bibliográfico. Uno de los sistemas de clasificación de la gravedad de estas complicaciones más usados es el de Clavien y Dindo, el cual permite estandarizar los términos. Las principales complicaciones de la cirugía laparoscópica son las lesiones vasculares e intestinales, pero también suelen presentarse lesiones vesiculares y uretrales, hernias e infecciones, entre otras de menor gravedad y frecuencia. En conclusión, es fundamental la búsqueda del perfeccionamiento de las técnicas existentes, estudios que garanticen su inocuidad y la creación de nuevas técnicas que disminuyan cada día más el número de complicaciones presentadas y se convierta en un procedimiento cada vez más seguro.

Palabras claves: Complicaciones; Cirugía; Laparoscopia; Procedimiento; Lesiones. 


\title{
Complicaciones de la cirugía laparoscópica
}

Vol. 3, núm. 2., (2019)

Adrián David Campoverde Cárdenas; Susy Arelis López Laaz; Wilson Pascual Correa Quinto; Jorge Daniel Cárdenas Rodríguez

\begin{abstract}
Laparoscopic surgery is today one of the most widely used procedures worldwide for its many benefits, however, this procedure is not exempt from complications. The main objective of this study is to capture the most important complications of laparoscopic surgery. The research design that was carried out is documentary or bibliographic. One of the classification systems of the severity of these most used complications is that of Clavien and Dindo, which allows the terms to be standardized. The main complications of laparoscopic surgery are vascular and intestinal lesions, but vesicular and urethral lesions, hernias and infections usually occur, among others of lesser severity and frequency. In conclusion, the search for the improvement of existing techniques, studies that guarantee their safety and the creation of new techniques that reduce the number of complications presented and become an increasingly safe procedure is essential.
\end{abstract}

Key words: Complications; Surgery; Laparoscopy; Procedure; Injuries. 


\section{Complicaciones de la cirugía laparoscópica}

Vol. 3, núm. 2., (2019)

Adrián David Campoverde Cárdenas; Susy Arelis López Laaz; Wilson Pascual Correa Quinto; Jorge Daniel Cárdenas Rodríguez

\section{Introducción.}

Las innovaciones continúas en el mundo de la cirugía médica, cada día incorpora nuevas técnicas con menor invasión, resultan menos agresivas y con mayores beneficios para los pacientes, tanto para el diagnóstico como para el tratamiento de enfermedades. Uno de estos tipos de cirugías mínimamente invasivas y de mayor uso a nivel mundial está constituido por la Laparoscopia.

Acerca del significado del término, García, Gutiérrez, \& Cueto (2016) explican que la raíz griega láparos se traduce como suave o flojo, posteriormente esta palabra cambió a lápara que se refería a los flancos del abdomen, es probable que por la blandura de los mismos. Más tarde, el uso evolucionó para llegar finalmente al significado de abdomen. Por otra parte, el verbo griego skopó significa mirar u observar. De esta combinación de los dos vocablos se origina la palabra laparoscopía, que significa ver dentro del abdomen. (p. 93)

Este concepto evolucionó conjuntamente con las técnicas y los diferentes usos que se le ha dado a la cirugía laparoscópica hasta la actualidad. En la actualidad se utiliza tanto para la exploración y diagnóstico como para el tratamiento de enfermedades.

El procedimiento quirúrgico laparoscópico utiliza un tubo bastante delgado llamado laparoscopio, este instrumento se inserta en el abdomen por medio de una pequeña incisión. Este tubo se caracteriza por tener una cámara conectada por medio de la cual se envían señales a un monitor de video, que permite al cirujano observar el interior del cuerpo, causando daños mínimos, de allí el término equivalente de cirugía mínimamente invasiva. Dentro de sus ventajas 


\section{Complicaciones de la cirugía laparoscópica}

Vol. 3, núm. 2., (2019)

Adrián David Campoverde Cárdenas; Susy Arelis López Laaz; Wilson Pascual Correa Quinto; Jorge Daniel Cárdenas Rodríguez

con respecto a la cirugía abierta se encuentran: la mínima permanencia del paciente en el centro hospitalario, mayor rapidez en la recuperación, menor dolor y minimizar las cicatrices. (Biblioteca Nacional de Medicina de los Estados Unidos, 2019)

Asimismo, para la laparoscopia usada en el tratamiento de enfermedades se lleva a cabo por medio de una o más incisiones pequeñas, por medio de las cuales se introducen al cuerpo tubos pequeños, y cámaras e instrumentos quirúrgicos diminutos. Estas cirugías mínimamente invasivas son menos riesgosas en comparación con una cirugía abierta, no obstante, existen riesgos de complicaciones relacionados con la anestesia, lesión de vasos u órganos, sangrado e infecciones. (Mayo Clinic, 2017)

Andrade et al, citado en Salazar, Ducasses, Domínguez, Mirabal, \& Rodríguez (2015) acerca de los bajos porcentajes de complicaciones, refieren que, en una revisión de 168 procedimientos llevados a cabo en un período de tres años, fueron informados un total de 9,5\% de complicaciones. Asimismo, cita a Escobar et al, quienes, en un estudio de 1.030 procedimientos urológicos laparoscópicos, encontraron un total de 103 complicaciones. Por último, citan a Castillo y Cortés, los cuales estudiaron un total de 1.924 cirugías urológicas realizadas tanto por vía lumboscópica como laparoscópica, donde encontraron una tasa de complicaciones de $22,4 \%$. (p. 1253)

En consecuencia, la cirugía laparoscópica es uno de los procedimientos más usados en la actualidad en todo el mundo, por sus múltiples beneficios, no obstante, no está exento de presentar algunas complicaciones. Dentro de las principales tenemos las lesiones vasculares e 


\section{Complicaciones de la cirugía laparoscópica}

Vol. 3, núm. 2., (2019)

Adrián David Campoverde Cárdenas; Susy Arelis López Laaz; Wilson Pascual Correa Quinto; Jorge Daniel Cárdenas Rodríguez

intestinales y menos frecuentes lesiones vesicales, uretrales, hernias, infecciones e incluso el cambio de laparoscopia a laparatomía.

La presente investigación revisa y plasma la descripción y algunas estadísticas de las principales complicaciones de la cirugía laparoscópica, así como su clasificación y los factores de riesgo que inciden en su aparición.

\section{Materiales y Métodos.}

La presente investigación se desarrolló mediante el uso de computadores personales con conexión a internet, a los fines de exponer todo lo relacionado con las complicaciones de la cirugía laparoscópica, es decir, que el mismo se enmarca en un diseño de investigación documental o bibliográfica.

Esta investigación se limita a la búsqueda y revisión sistemática de material bibliográfico accesible mediante el uso de diversas bases de datos, entre las que figuraron: MedlinePlus, PubMed, Biblioteca Virtual de la Salud (BVS), SciELO, Dialnet y ELSEVIER, entre otras; mediante las cuales se sintetizó la mejor evidencia disponible.

Se realizó una búsqueda aleatoria y consecutiva, usando las expresiones "Complicaciones de la cirugía laparoscópica", "Cirugía laparoscópica"; "Laparoscopia” y "Cirugía mínimamente invasiva", lo que aproximadamente resultó en más de un centenar de miles de registros bibliográficos, los cuales se filtraron bajo los criterios de idioma español, relevancia, correlación temática y fecha de publicación en los últimos cinco años, sin descartar por tipo de material bibliográfico. 


\section{Complicaciones de la cirugía laparoscópica}

Vol. 3, núm. 2., (2019)

Adrián David Campoverde Cárdenas; Susy Arelis López Laaz; Wilson Pascual Correa Quinto; Jorge Daniel Cárdenas Rodríguez

Por último, se leyó y analizó la evidencia científica recabada, resultando el contenido de la presente investigación.

\section{Resultados.}

Sistema de Clasificación de Complicaciones Intraoperatorias y Postquirúrgicas Tempranas

Salazar et al. (2015) acerca de la clasificación de las complicaciones de la cirugía laparoscópica, refieren que, en el año 1.992, Clavien y Dindo propusieron un sistema que fue aceptado y revisado en 2.004, que permite estandarizar los términos en cuanto a las complicaciones posquirúrgicas. Este sistema consta de 5 grados: Grado I: aquellas que ocasionan desviación del curso normal del período posoperatorio, sin embargo, no requiere una intervención quirúrgica, endoscópica o radiológica; sólo requiere la administración de medicamentos básicos. Grado II: estos casos requieren atención farmacológica, nutrición parenteral total o transfusión. Grado III: estas complicaciones requieren intervención quirúrgica, radiológica o endoscópica. Grado IV: estas complicaciones son aquellas que requieren que el paciente sea internado en una Unidad de Cuidados Intensivos. Grado V: es el grado más grave de las complicaciones ya que ocasionan la muerte. (p. 1250)

\section{Complicaciones de la cirugía laparoscópica}

Por lo general, las complicaciones de la cirugía laparoscópica van a depender en gran medida del grado de complejidad del procedimiento quirúrgico. Asimismo, la experiencia del cirujano es un factor que influye en la aparición de complicaciones. Estas complicaciones pueden 


\section{Complicaciones de la cirugía laparoscópica}

Vol. 3, núm. 2., (2019)

Adrián David Campoverde Cárdenas; Susy Arelis López Laaz; Wilson Pascual Correa Quinto; Jorge Daniel Cárdenas Rodríguez

aparecer durante la cirugía o postoperatoriamente, incluso pueden influir en el cambio de cirugía para lograr el tratamiento inicial, en la programación de una nueva cirugía para corregir la complicación y, en el peor de los casos, y con un porcentaje mínimo, la muerte.

Por otra parte, existen factores de riesgo en el paciente que predisponen la aparición de complicaciones en la cirugía laparoscópica, los cuales se expondrán posteriormente.

Las lesiones más comunes en la cirugía laparoscópica son las lesiones vasculares e intestinales, pero también suelen presentarse lesiones vesiculares y uretrales, hernias e infecciones, entre otras de menor gravedad.

\section{Lesiones vasculares.}

Recari, Oroz, \& Lara (2009) Uno de los daños vasculares más frecuentes ocurre por laceración de vasos mesentéricos, consecuencia de la introducción de la aguja de Veress por medio del ombligo, especialmente si hay adherencias de epiplon a este nivel. Cuando la aguja de Veress se introduce en un vaso de gran calibre, este hecho se detecta con facilidad por la salida de sangre que se detecta a través de la misma, o en la primera maniobra de comprobación. Por otra parte, la insuflación directa de $\mathrm{CO} 2$ al torrente sanguíneo es capaz de terminar con una embolia gaseosa. Por otra parte, existen lesiones vasculares producidas por los trocares, los cuales son capaces de punzar grandes vasos abdominales, tales como la aorta, mesentérica inferior o cava. Esta complicación ocurre con la introducción del primer trócar transumbilical la cual no es superior al 0,3-0,9\% y existe mayor probabilidad en aquellos casos de pacientes muy delgadas o niñas, donde es fundamental tener en cuenta la posición de los grandes vasos sobre la 


\section{Complicaciones de la cirugía laparoscópica}

Vol. 3, núm. 2., (2019)

Adrián David Campoverde Cárdenas; Susy Arelis López Laaz; Wilson Pascual Correa Quinto; Jorge Daniel Cárdenas Rodríguez

columna, los cuales se encuentran tan sólo a un tercio de profundidad del total del grosor externo del tronco. (p. 74)

Con respecto a las complicaciones intraoperatorias, puede encontrarse sangrado de la vena renal y del lecho quirúrgico en el transcurso de una nefrectomía laparoscópica, el cual se evidencia en un estudio de Salazar et al. (2015), producido por causa de la ligadura de la vena renal con clips metálicos los cuales fueron insuficientes para abarcar con un margen de seguridad el diámetro de dicha vena. La resolución de la complicación se realizó por medio de un cambio de procedimiento de cirugía laparoscópica a cirugía abierta. Asimismo, refieren que, en publicaciones relacionadas con este mismo tema, se ha descrito la misma lesión en un rango de 0 a $5 \%$. En otros estudios de complicaciones vasculares, los autores citan a Andrade et al. Los cuales en su estudio presentaron una tasa de complicaciones vasculares de 4,7\%. (p. 1253)

Para Sepúlveda-Agudelo (2011) las complicaciones vasculares suelen estar relacionadas con la técnica de entrada del procedimiento laparoscópico. En cuanto a la incidencia mencionan en su trabajo que esta va de $0,01 \%$ a $0,64 \%$, no obstante, son severas. Estas presentan una tasa de mortalidad del $9 \%$ y 17\%. Los vasos de mayor riesgo por su posición anatómico durante la entrada de la laparoscopia son la arteria aorta, los vasos iliacos y la vena cava inferior. El vaso que presenta mayor compromiso durante estos procedimientos es la arteria epigástrica inferior, y viene dada frecuentemente por inserción de trócares. (p. 89) 


\section{Complicaciones de la cirugía laparoscópica}

Vol. 3, núm. 2., (2019)

Adrián David Campoverde Cárdenas; Susy Arelis López Laaz; Wilson Pascual Correa Quinto; Jorge Daniel Cárdenas Rodríguez

\section{Lesiones Intestinales.}

Las lesiones intestinales constituyen más de la mitad de las complicaciones mayores en la cirugía laparoscópica. Esta complicación presenta una incidencia de 0,06\% a 0,65\%. Puede existir sospecha de la misma cuando se aspira el contenido fecal, caracterizado por presencia de olor fecaloide o por una presión a la entrada entre 8 y 10 milímetros de mercurio (mm $\mathrm{Hg}$ ). (Sepúlveda-Agudelo, 2011, p. 89,90)

Ciertamente, constituyen una de las complicaciones más serias, ya que puede ser diagnosticada postquirúrgicamente, lo que aumenta el riesgo de peritonitis fecal y muerte. Las lesiones de intestino grueso son una complicación muy rara, se presenta con un porcentaje del $0,1 \%$ y su presencia afecta al colon descendente. Uno de los principales síntomas, es el olor fétido, así como la visualización de la luz intestinal. Un diagnóstico tardío presenta una clínica con cuadro de dolor abdominal y contractura de abdomen. Es probable que el paciente requiera laparatomía para llegar al diagnóstico, con una cobertura antibiótica previa. "La reparación o resección debe ir seguida de una ileostomía si la lesión afecta al colon ascendente, o de una colostomía en las demás localizaciones. El cierre debe hacerse dejando drenajes”. (Recari, Oroz, \& Lara, 2009, p. 75)

En el estudio de Salazar et al. (2015) relacionado con las complicaciones posquirúrgicas tempranas de la laparoscopia, se presentó una perforación de colon como complicación de una nefrectomía, la cual se identificó en el período posoperatorio mediato, requiriendo una colostomía por medio de cirugía abierta. Esta complicación representó $1 \%$ del total, tasa similar a la notificada en otros estudios. (p. 1253) 


\section{Complicaciones de la cirugía laparoscópica}

Vol. 3, núm. 2., (2019)

Adrián David Campoverde Cárdenas; Susy Arelis López Laaz; Wilson Pascual Correa Quinto; Jorge Daniel Cárdenas Rodríguez

Lesiones vesicales.

Es otro tipo de lesión grave, aunque infrecuente. Una punción vesical que pasa inadvertida podría generar lesiones graves, sobre todo en aquellos casos que se produce extra peritonealmente. En caso de detectarla, se debe llevar a cabo "una sutura inmediata de la brecha y la colocación de una sonda permanente, como mínimo seis días, bajo protección antibiótica". Caso contrario, si la lesión pasa inadvertida, observándose en el postoperatorio, se recomienda una nueva exploración laparoscópica a los fines de visualizar el orificio vesical que comunica con el peritoneo y su sutura. (Recari et al., 2009, p. 75)

\section{Lesiones uretrales.}

Estas lesiones propias de la cirugía laparoscópica, se incrementaron en frecuencia con la llegada de dicho abordaje. El principio básico en la cirugía laparoscópica adyacente a las estructuras uretrales, es la identificación de su trayecto antes de proceder. Presentan mayor exposición durante los procedimientos de histerectomía total laparoscópica, la enfermedad inflamatoria pélvica y la endometriosis. Asimismo, existen factores que predisponen las complicaciones uretrales, como los tumores pélvicos, los cuales al alteran la anatomía. "Los tres lugares más comunes son, en orden de frecuencia: a nivel de infundíbulo-pélvico, en el cruce a nivel de arteria uterina en el parametrio y en su entrada a la vejiga”. (Recari et al., 2009, p.76,77).

El reporte de incidencia de las lesiones de vejiga y uréteres es de $0,03 \%$ a $0,13 \%$, de las cuales las lesiones de la vejiga representan las de mayor frecuencia, de 2 a 3 veces, en 


\section{Complicaciones de la cirugía laparoscópica}

Vol. 3, núm. 2., (2019)

Adrián David Campoverde Cárdenas; Susy Arelis López Laaz; Wilson Pascual Correa Quinto; Jorge Daniel Cárdenas Rodríguez

comparación con las lesiones ureterales. "Los tipos de lesiones urinarias más comunes son perforación vesical, seguida de fístula, ligadura de uréter y corte de uréter; sin embargo, no se ha reportado diferencia con la cirugía abierta ginecológica”. Los principales síntomas que generan sospecha de lesión vesical son vómito, fiebre, dolor o distensión abdominal, escalofrío, oliguria, retención urinaria, anuria o goteo persistente de orina. (Sepúlveda-Agudelo, 2011, p. 90)

\section{Hernias.}

La aparición de hernia intestinal por medio de los defectos de pared, son de mayor frecuencia en los casos de procedimientos que ameritan múltiples entradas, el uso de dilatadores que aumentan el daño de la fascia o la extracción de grandes tumores. La Asociación Americana de Laparoscopistas Ginecológicos en un estudio de 4.385 procedimientos laparoscópicos, recogía 933 hernias, de las cuales un $80 \%$ se presentaban a pesar del cierre de la fascia. Asimismo, el estudio estableció que el riesgo es mayor en los sitios extraumbilicales y aumentan con el uso de trócares de $12 \mathrm{~mm}(3,1 \%)$ en comparación con los trocales de $10 \mathrm{~mm}(0,23 \%)$, razón por la cual la fascia debería cerrarse en estas incisiones. (Recari et al., 2009, p. 75)

Baltodano \& Solís, (2017) acotan que la formación de hernias postneumoperitoneo es infrecuente y rara. Al respecto, se han documentado casos de pacientes tratados con laparoscopia: colecistectomía, fundoplicatura, apendicectomía, y donde se utilizó una presión de neumoperitoneo variable de 14 a $16 \mathrm{mmHg}$ durante el procedimiento, no obstante, en el transcurso de unos días se desarrolló una hernia inguinal. En consecuencia, la causa del desarrollo de hernias postquirúrgicos se debe a factores tales como un saco preformado, la 


\section{Complicaciones de la cirugía laparoscópica}

Vol. 3, núm. 2., (2019)

Adrián David Campoverde Cárdenas; Susy Arelis López Laaz; Wilson Pascual Correa Quinto; Jorge Daniel Cárdenas Rodríguez

posición durante la cirugía, alta presión de neumoperitoneo y uso de fármacos anestésicos. (p. 71-72)

Necesidad de que le hagan una laparotomía.

Es un cambio de modalidad quirúrgica, sin programación previa, que va de realizar una cirugía laparoscópica a una laparotomía, y cuya decisión imprevista obedece a que el primer procedimiento quirúrgico programado originalmente no se pudo hacer con las incisiones más pequeñas, propias de la laparoscopia, y la condición encontrada del paciente requirió de una incisión mayor para llevar a cabo el tratamiento con éxito.

\section{Otras complicaciones.}

Las infecciones son una de las complicaciones más infrecuentes, representan el $0,1 \%$ de los casos, aproximadamente. Otras complicaciones infrecuentes son los hematomas y la formación de adherencias. "Además, se pueden presentar lesiones neurológicas ocasionadas básicamente por mala posición de la paciente durante la cirugía”. (Sepúlveda-Agudelo, 2011, p. 92)

Comorbilidad del paciente que influye en las complicaciones de la cirugía laparoscópica (factores de riesgo).

Díaz, Aguirre, García, \& Castillo (2018) aseveran que existen factores de riesgo importantes que predisponen la aparición de complicaciones en la cirugía laparoscópica, que son propios de la condición del paciente previo y posterior a la intervención, tales como anemia 


\section{Complicaciones de la cirugía laparoscópica}

Vol. 3, núm. 2., (2019)

Adrián David Campoverde Cárdenas; Susy Arelis López Laaz; Wilson Pascual Correa Quinto; Jorge Daniel Cárdenas Rodríguez

crónica preoperatoria, desnutrición, historia de consumo de drogas, tabaquismo, necesidad de exploración de vía biliar, infección previa por Staphylococcus aureus, neumonía o infección de vías urinarias en el periodo postquirúrgico. (p. 148)

Para Bocanegra \& Córdova (2013) conforme aumenta la edad del paciente aumenta el número de patologías asociadas, además de complicaciones, razón por la cual, la edad avanzada representa un factor de riesgo de mortalidad, conversiones intraoperatorias a colecistectomía abierta y complicaciones intra o postoperatorias. Esto ocasiona desconcierto y mantiene al personal cirujano alerta en la toma de decisiones para la obtención de los mejores resultados. (p. 114)

Para Saborit, Ortíz, Rosabal, Ramos, \& Capote (2019) la edad también representa un factor de riesgo para la aparición de complicaciones en la laparoscopia, siendo la población más vulnerable a estas complicaciones los niños y los ancianos. La colecistectomía laparoscópica, es una de las intervenciones quirúrgicas más comunes practicadas en adultos mayores. El tabaquismo, los pacientes con edades de 75 años o mayores y el alcoholismo constituyen factores de riesgo quirúrgico relacionados con la aparición de complicaciones anestésicas, en pacientes geriátricos, durante colecistectomía video laparoscópica. (p. 26)

\section{Conclusión.}

La cirugía laparoscópica, debido a sus ventajas, se ha convertido hoy en día, en la vía de elección para la exploración, diagnóstico y tratamiento de innumerables afecciones. Este tipo de cirugía mínimamente invasiva, se ha convertido en la más usada a nivel mundial por sus 


\section{Complicaciones de la cirugía laparoscópica}

Vol. 3, núm. 2., (2019)

Adrián David Campoverde Cárdenas; Susy Arelis López Laaz; Wilson Pascual Correa Quinto; Jorge Daniel Cárdenas Rodríguez

múltiples beneficios, entre los que destacan menores complicaciones, no obstante, no se encuentra exenta de presentarlas.

La baja incidencia de los casos de complicaciones de las cirugías laparoscópicas en todo el mundo podría sugerir una escaza importancia del problema, no obstante, los principales problemas no se centran en la incidencia, la cual es muy baja, sino en que es una de las cirugías más practicadas a nivel mundial y sus principales complicaciones, sobre todo las más frecuentes, son muy graves y en muchos casos terminan en la muerte del paciente.

El avance de la tecnología y las nuevas técnicas para la entrada en cirugía laparoscópica, hacen imperiosa la realización de nuevas investigaciones, se requieren estudios para evaluar las nuevas técnicas ya que no existen evidencias que garanticen los beneficios de su uso y es imperioso que estas soporten un menor riesgo de complicaciones, especialmente de lesiones vasculares e intestinales.

A pesar de la baja incidencia de casos de complicaciones de cirugía laparoscópica en comparación con la presentada por la cirugía tradicional, es fundamental la búsqueda del perfeccionamiento de las técnicas existentes, estudios que garanticen su inocuidad y la creación de nuevas técnicas que disminuyan cada día más el número de complicaciones presentadas y se convierta en un procedimiento cada vez más seguro. 


\section{Complicaciones de la cirugía laparoscópica}

Vol. 3, núm. 2., (2019)

Adrián David Campoverde Cárdenas; Susy Arelis López Laaz; Wilson Pascual Correa Quinto; Jorge Daniel Cárdenas Rodríguez

\section{Bibliografía.}

Baltodano, A., \& Solís, J. (2017). Hernia femoral como complicación posterior a una colecistectomía laparoscópica. Acta Médica Costarricense, 59(2), 70-72. Recuperado el 05 de Agosto de 2019, de https://www.scielo.sa.cr/scielo.php?script=sci_arttext\&pid=S0001-60022017000200070

Biblioteca Nacional de Medicina de los Estados Unidos. (12 de Marzo de 2019). MedlinePus. Recuperado el 03 de Agosto de 2019, de https://medlineplus.gov/spanish/pruebas-delaboratorio/laparoscopia/

Bocanegra, R., \& Córdova, M. (2013). Colecistectomía laparoscópica en el adulto mayor: complicaciones postoperatorias en mayores de 75 años en el Hospital Nacional Cayetano Heredia, Lima, Perú, del 2007 - 2011. Sociedad de Gastroenterología del Perú, 33(2), 113-120. Recuperado el 06 de Agosto de 2019, de http://www.scielo.org.pe/pdf/rgp/v33n2/a03v33n2.pdf

Díaz, J., Aguirre, I., García, R., \& Castillo, A. R. (31 de Julio de 2018). Complicaciones asociadas a colecistectomía laparoscópica electiva. Salud Jalisco, 5(5), 144-149. Recuperado el 06 de Agosto de 2019, de https://www.medigraphic.com/pdfs/saljalisco/sj2018/sj183e.pdf

García, A., Gutiérrez, L., \& Cueto, J. (Abril-Junio de 2016). Evolución histórica de la cirugía laparoscópica. Cirugía Endoscópica, 17(2), 93-106. Recuperado el 05 de Agosto de 2019, de https://www.medigraphic.com/pdfs/endosco/ce-2016/ce162i.pdf

Mayo Clinic. (17 de Diciembre de 2017). mayoclinic.org. Recuperado el 06 de Agosto de 2019, de https://www.mayoclinic.org/es-es/tests-procedures/minimally-invasivesurgery/about/pac-20384771

Recari, E., Oroz, L., \& Lara, J. (2009). Complicaciones de la cirugía ginecológica. An. Sist. Sanit. Navar., 32(1), 65-79. Recuperado el 06 de Agosto de 2019, de http://scielo.isciii.es/pdf/asisna/v32s1/ginecologia7.pdf

Saborit, L., Ortíz, C., Rosabal, M., Ramos, A., \& Capote, G. (2019). Factores pronóstico de morbilidad anestésica en el paciente geriátrico. Anestesia en México, 31(1), 13-29. Recuperado el 05 de Agosto de 2019, de http://www.scielo.org.mx/pdf/am/v31n1/24488771-am-31-01-13.pdf

Salazar, A., Ducasses, S., Domínguez, E., Mirabal, A., \& Rodríguez, A. (26 de Septiembre de 2015). Complicaciones intraoperatorias y posquirúrgicas tempranas en cirugía lumbolaparoscópica urológica. MEDISAN, 19(10), 1248-1258. Recuperado el 03 de Agosto de 2019, de http://scielo.sld.cu/pdf/san/v19n10/san101910.pdf 


\section{Complicaciones de la cirugía laparoscópica}

Vol. 3, núm. 2., (2019)

Adrián David Campoverde Cárdenas; Susy Arelis López Laaz; Wilson Pascual Correa Quinto; Jorge Daniel Cárdenas Rodríguez

Sepúlveda-Agudelo, J. (2011). Complicaciones laparoscópicas asociadas a la técnica de entrada. Revista Colombiana de Obstetricia y Ginecología, 62(1), 88-93. Recuperado el 06 de Agosto de 2019, de http://www.scielo.org.co/pdf/rcog/v62n1/v62n1a11.pdf

$$
\text { (c) }(1)(2)(2)
$$

RECONOCIMIENTO-NOCOMERCIAL-COMPARTIRIGUAL

CC BY-NC-SA

ESTA LICENCIA PERMITE A OTROS ENTREMEZCLAR, AJUSTAR Y CONSTRUIR A PARTIR DE SU OBRA CON FINES NO COMERCIALES, SIEMPRE Y CUANDO LE RECONOZCAN LA AUTORÍA Y SUS NUEVAS CREACIONES ESTÉN BAJO UNA LICENCIA CON LOS MISMOS TÉRMINOS. 